\title{
Measurement of serum suPAR is not ready for clinical
} use

\section{Jeroen Deegens and Jack Wetzels}

We thank Howard Trachtman and Jochen Reiser for their comment (suPAR is the circulating factor in some but not all FSGS. Nat. Rev. Nephrol. 26 August 2014; doi:10.1038/nrneph.2014.113-c1 $)^{1}$ on our News \& Views commentary (The search goes on: suPAR is not the elusive FSGS factor. Nat. Rev. Nephrol. 10, 431-432; 2014). ${ }^{2}$ Trachtman and Reiser use the football World Cup as a metaphor for scientific research. In this respect, we wonder what is a goal? For scientists a high-impact publication or grant approval might be considered a goal, whereas for clinicians and patients the score is opened if research leads to direct patient benefit. Currently the Tour de France is receiving attention. We feel that patient-centred research is best compared with a team time trial in a mountainous region; close collaboration is needed and each member takes the lead for shorter or longer time periods. Moreover, when the top of a mountain is reached the view is spectacular, but new peaks are visible on the horizon and the finish remains out of sight.

Reiser and his group have performed important experimental studies in mice and podocytes that have provided evidence for a possible role of soluble urokinase plasminogen activator receptor (suPAR) in focal segmental glomerulosclerosis (FSGS). ${ }^{3}$ However, when writing a commentary on new data, ${ }^{2}$ we believe that it is our task as clinicians and clinical researchers to judge if scientific findings can be directly applied to the patients we look after. In their study, Reiser and colleagues validated their findings in patients with FSGS. ${ }^{3}$ Based on these results, many patients and physicians thought that measurement of suPAR could immediately be applied in patient care. Unfortunately, the study included a heterogeneous population with poorly phenotyped patients and a lack of appropriate controls. Indeed, recent clinical studies showed that when adjusted for estimated glomerular filtration rate (eGFR), levels of intact suPAR do not differentiate between patients with idiopathic FSGS and those with secondary FSGS, or between patients with FSGS, other glomerular diseases or chronic kidney disease. ${ }^{4-8}$ In our commentary we concluded that no role exists for the measurement of intact suPAR levels in patients with FSGS. Unglycosylated suPAR or suPAR fragments remain important candidates for the circulating permeability factor in this disease, but further studies are needed to provide proof of concept.

We are not convinced that the study by Alachkar et al. demonstrates that levels of intact suPAR correlate with recurrent FSGS. ${ }^{9}$ Their retrospective analysis included patients with de novo FSGS, and recurrent FSGS was diagnosed in patients with foot process effacement (FPE) of only $13 \%$ and $1 \mathrm{~g} / \mathrm{g}$ proteinuria. The authors reported significantly higher suPAR levels in patients with severe FPE $(n=4)$ than in those with mild FPE $(n=5)$, independent of eGFR. However, median eGFR was $22 \mathrm{ml} / \mathrm{min}$ in the severe FPE group versus $38 \mathrm{ml} / \mathrm{min}$ in the mild FPE group, and with such small numbers of patients the power to detect significant differences is low. After treatment with plasmapheresis, suPAR levels decreased significantly. However, suPAR data were available for only seven of 24 patients and individual clinical data were not provided. Of note, eGFR improved significantly after treatment in all 24 patients.

The observed association between suPAR levels and complications in patients with type 1 diabetes mellitus is interesting, although the authors admit that these data do not prove causality. ${ }^{10,11}$ Particularly interesting is data from Yoo et al. that show similar suPAR levels in patients with highrisk FSGS and in patients with diabetes and macroalbuminuria. ${ }^{10}$ This finding further proves that levels of suPAR, as measured by current technology, are unable to differentiate between FSGS and other kidney diseases.

Reiser and colleagues must receive credit for their experimental research directed at finding the important FSGS factor. suPAR is an intriguing protein and potentially involved in human disease. Unfortunately, its role in FSGS recurrence is not firmly established and the new data discussed above do not contradict our conclusion that measurement of intact suPAR is not useful in patients with FSGS. ${ }^{2}$

Department of Nephrology, Radboud University Medical Centre, PO Box 9101, 6500 HB Nijmegen, Netherlands (J.K.D., J.F.W.).

Correspondence to: J.W.

jack.wetzels@radboudumc.nl

Competing interests

The authors declare no competing interests.

1. Trachtman, H. \& Reiser, J. suPAR is the circulating factor in some but not all FSGS. Nat. Rev. Nephrol. http://dx.doi.org/10.1038/ nrneph.2014.113-c1.

2. Deegens, J. K. \& Wetzels, J. F. The search goes on: suPAR is not the elusive FSGS factor. Nat. Rev. Nephrol. 10, 431-432 (2014).

3. Wei, C. et al. Circulating urokinase receptor as a cause of focal segmental glomerulosclerosis. Nat. Med. 17, 952-960 (2011).

4. Meijers, B. et al. The soluble urokinase receptor is not a clinical marker for focal segmental glomerulosclerosis. Kidney Int. 85, 636-640 (2014).

5. Bock, M. E., Price, H. E., Gallon, L. \& Langman, C. B. Serum soluble urokinase-type plasminogen activator receptor levels and idiopathic FSGS in children: a single-center report. Clin. J. Am. Soc. Nephrol. 8, 1304-1311 (2013).

6. Sinha, A. et al. Serum-soluble urokinase receptor levels do not distinguish focal segmental glomerulosclerosis from other causes of nephrotic syndrome in children. Kidney Int. 85, 649-658 (2014).

7. Wada, T. et al. A multicenter cross-sectional study of circulating soluble urokinase receptor in Japanese patients with glomerular disease. Kidney Int. 85, 641-648 (2014).

8. Maas, R. J., Wetzels, J. F. \& Deegens, J. K. Serum-soluble urokinase receptor concentration in primary FSGS. Kidney Int. 81, 1043-1044 (2012). 


\section{CORRESPONDENCE}

9. Alachkar, N. et al. Podocyte effacement closely links to suPAR levels at time of posttransplantation focal segmental glomerulosclerosis occurrence and improves with therapy. Transplantation 96, 649-656 (2013).
10. Yoo, T. H. et al. Sphingomyelinase-like phosphodiesterase $3 \mathrm{~b}$ expression levels determine podocyte injury phenotypes in glomerular disease. J. Am. Soc. Nephrol. http:// dx.doi.org/10.1681/ASN.2013111213.
11. Theilade, S. et al. Soluble urokinase plasminogen activator receptor levels are elevated and associated with complications in patients with type 1 diabetes. J. Intern. Med. http://dx.doi.org/10.1111/joim.12269. 